\title{
PLACENTAL MESENCHYMAL DYSPLASIA, A RARE CAUSE OF NON MOLAR PLACENTAL HYDROPS: A CASE REPORT
}

Ramya ${ }^{1}$, Uma Maheshwari G², Chaitra $V^{3}$

\section{HOW TO CITE THIS ARTICLE:}

Ramya T, Uma Maheshwari G, Chaitra V. "Placental Mesenchymal Dysplasia, a Rare Cause of Non- Molar Placental Hydrops: A Case Report". Journal of Evolution of Medical and Dental Sciences 2014; Vol. 3, Issue 64, November 24; Page: 14110-14113, DOI: 10.14260/jemds/2014/3889

ABSTRACT: Placental mesenchymal dysplasia (PMD) is a rare, incompletely understood placental vascular lesion. Ultrasound and gross placental examination reveal placentomegaly and grape like vesicles that mimic molar pregnancy. PMD is often associated with normal foetus, diploid karyotyping and needs to be differentiated from its mimickers as the prognosis and management varies. Here in, we describe a case of PMD with ultrasound and histopathological correlation.

KEYWORDS: Placental mesenchymal dysplasia, molar pregnancy, diploid karyotyping.

INTRODUCTION: Placental mesenchymal dysplasia (PMD), is considered as placental vascular anomaly with diffuse mesenchymal stem villous hyperplasia and was first described by Moscoso et al. in 1991. ${ }^{1}$ The exact incidence of PMD is unknown, some of the studies show the incidence to be $0.02 \% .^{2}$ PMD is characterized by enlarged placenta with dilated, fluid filled vesicles. Prenatal ultrasonography reveals an enlarged, thickened placenta with cystic spaces in the sub chorionic zone. Hence, PMD has also been referred to as "pseudo- partial" mole or non-molar placental hydrops.3,4,5,6

CASE REPORT: A 28 year old woman, G2P1L1, known case of rheumatic heart disease, post aortic valve replacement with mechanical prosthesis and on warfarin, was referred to our hospital, at 21 weeks of gestation. Her previous child birth was 9 months back and she was unaware of the present pregnancy till $20^{\text {th }}$ week of gestation. On admission, patient was severely anaemic and in heart failure. Ultrasound examination showed a single live intrauterine foetus with no structural abnormalities.

Placenta was anterior, diffusely enlarged with few isoechoic retro placental collection, the largest measured $12 \times 3.9 \mathrm{~cm}$ (Figure: 1). There was no colour uptake in the isoechoic areas. Subchorionic zone contained few hypoechoic cysts (Figure: 2). The size of the placenta was large when compared with that of the foetus (Figure: 3). Lower end of placenta was seen covering the internal os. Umbilical and uterine artery doppler studies were normal.

As there was no improvement in haemoglobin levels even after two packed cell transfusions and repeat USG showed increasing retroplacental clots, abruptio placenta was suspected and patient planned for hysterotomy. Repeat transfusions and prophylactic uterine artery embolization was done before proceeding with hysterotomy. Per operatively there was $650 \mathrm{gms}$ of retroplacental clots. The placenta was cut through and foetus removed. Uterus was atonic, hence B-Lynch suture applied and concurrent sterilization done. Patient was on supportive ventilation and later weaned uneventfully. As consent for foetal autopsy could not be obtained, only placenta was sent for histopathological examination.

Macroscopically, the placenta was large for gestational age, both in dimension and weight. The placenta weighed $650 \mathrm{gms}$ ( $>90^{\text {th }}$ percentile). There were a few vesicles in the parenchyma, largest measuring $1.0 \times 1.0 \times 0.5 \mathrm{~cm}$. The chorionic plate was not identified and there were thick ropy stem villi that have been shorn off the villous parenchyma (Figure 4). Chunks of blood clot were also present. Histopathological examination showed collapsed vesicles with central cystic degeneration 


\section{CASE REPORT}

and absent trophoblastic proliferation (Figure 5). Thin walled, dilated vascular spaces of variable sizes were present in the stem villi (Figure 6). Hydropic change and cellular islands were prominent. Hence a diagnosis of placental mesenchymal dysplasia was offered.

DISCUSSION: PMD is a rare benign condition that should be considered in the differential diagnosis when ultrasound findings demonstrate a placenta with cystic changes and a normal foetus. The exact incidence of PMD is unknown because of its rarity. It is also under-diagnosed and under-reported, as not all pathologists are aware of this entity. A strong female preponderance is noted with a ratio of 3.6-4.0:1(F: M ratio).

Although the exact etiology of PMD is unclear, there are multiple competing theories, including congenital malformation of the mesoderm, ${ }^{7}$ hypoxia and hypoperfusion of unknown aetiology, ${ }^{8}$ molecular disruption of the imprinting genes of chromosome 11p15.5 associated with Beckwith-Wiedemann syndrome ${ }^{4,9}$ and androgenetic/biparental mosaicism. ${ }^{10}$

The other causes of cystic placentas are partial molar pregnancy, complete mole with coexisting normal foetus, chorioangioma, subchorionic haematoma, confined placental mosaicism and spontaneous abortion with hydropic changes.

PMD is most often mistaken for molar pregnancy based on similar sonographic findings between the two entities-enlarged, thickened placenta with cystic spaces. ${ }^{11}$ Molar pregnancies always exhibit elevated hCG levels, while in one series of PMD by Nayeri et al. ${ }^{2} 38 \%$ of the cases showed elevated hCG level. In the present case hCG level was mildly elevated. PMD is more likely to be associated with elevated ms AFP levels. Invasive testing by chorionic villus sampling or amniocentesis should be performed to confirm a normal karyotype, thereby excluding partial molar pregnancy.

One-fourth to one-third cases of PMD is associated with Beckwith-Wiedeman syndrome ${ }^{10}$ (macrosomia, hemihyperplasia, visceromegaly, omphalocele, macroglossia, renal anomalies and adrenal cytomegaly). There are multiple case reports in the literature describing the co-existence of PMD with foetal hepatic mesenchymal tumors, suggesting a common pathogenetic origin for the two anomalies. ${ }^{12}$ Carta et al. ${ }^{12}$ proposed that the two conditions may arise from synchronous abnormal development of the mesoderm.

Pregnancy outcomes range from healthy, uncomplicated pregnancies to adverse maternal and/or neonatal complications. Only $9 \%$ of pregnancies affected by PMD are uncomplicated with regards to either neonatal or maternal complications. The most common adverse event is preterm labour with an incidence of $50 \%^{2}$ of which $33 \%$ were of spontaneous preterm labour. Other complications are IUGR (33\%), IUFD (13\%) and PPROM (17\%). Pham et al ${ }^{8}$ proposed that poor perinatal outcome may occur because of obstructive vascular thrombosis and decreased maternalfoetal gas exchange owing to a reduction in the normal amount of chorionic villi. The only maternal complication was hypertension which developed in $9 \%$ of cases. ${ }^{2}$

The diagnosis of PMD is only affirmed after pathological evaluation of placenta. Grossly, it is characterised by placentomegaly, dilated or even aneurysmal chorionic vessels and enlarged hydropic or cystic villi.1,13 Microscopic findings include enlarged, edematous stem villi with mesenchymal hyperplasia and cistern formation. Thick-walled blood vessels can be seen at the periphery of stem villi. A clinching point in diagnosing PMD is the absence of trophoblastic hyperplasia, which helps to differentiate it from gestational trophoblastic disease.1,11,13 The above mentioned findings were noted in our case, hence a diagnosis of PMD was offered. 


\section{CASE REPORT}

CONCLUSION: Placental mesenchymal dysplasia is a rare, unfamiliar placental pathology, characterized by placentomegaly with cystic change and can be mistaken for molar pregnancy. It should be considered as a differential diagnosis when the above findings are associated with a normal appearing foetus. Hence, a detailed clinical, ultrasonographical and histopathological examination along with a high index of suspicion is mandatory to arrive at a correct diagnosis of this entity thereby unnecessary termination of pregnancy can be avoided.

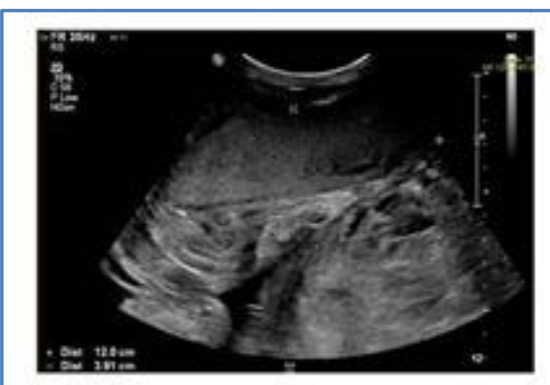

Fig-1 : Large retroplacental collection due to placental separation

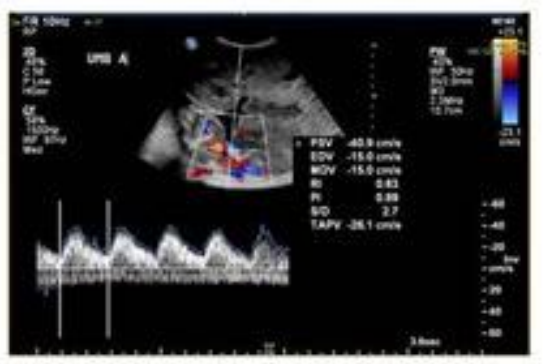

Fig-2 : Transabdominal ultrasound study showing placenta with multiple hypoechoic cysts

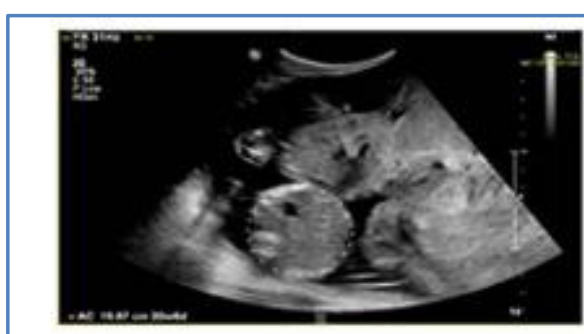

Fig-3 : Enlarged placenta The size of the placenta is appreciated when compared with the suze of the fetal abdomen

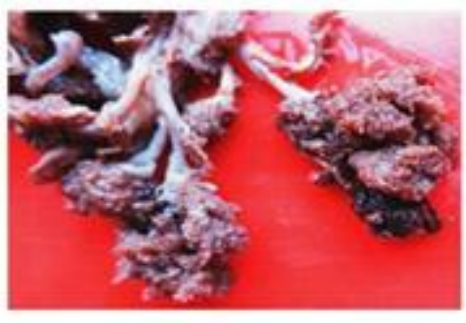

Fig-4:Thick, ropy stem villi that have been shorn off the villous parenchyma

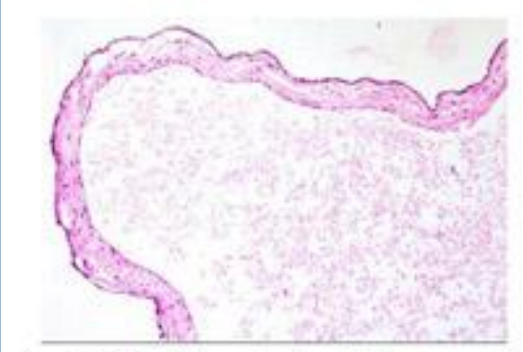

Fig-5: Villi with central cystic degeneration and no trophoblastic proliferation (10x)

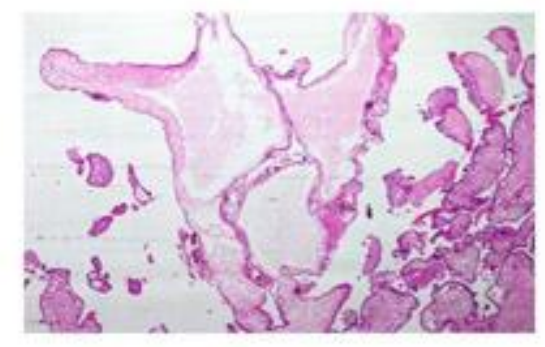

Fig-6: Stem villi with cistern formation (4X)

\section{REFERENCES:}

1. Moscoso G, Jauniax E, Hustin J. Placental vascular anomaly with diffuse mesenchymal stem villous hyperplasia: a new clinic-pathologic entity? Pathol Res Pract. 1991; 187: 324-328.

2. Umazume T, Kataoka S, Kamamuta K, et al. Placental mesenchymal dysplasia, a case of intrauterine sudden death of fetus with rupture of cirsoid periumbilical chorionic vessels. Diagn Pathol 2011; 6: 38.

3. Baergen RN. Manual of pathology of the human placenta. $2^{\text {nd }}$ edition. New York: Springer; 2011. P. 220-420. 
4. Pham T, Steele J, Stayboldt C, et al. Placental mesenchymal dysplasia is associated with high rates of intrauterine growth restriction and fetal demise: a report of11 new cases and review of literature. Am J Clinical Pathol 2006; 126: 67-78.

5. Paradinas FJ, Sebire NJ, Fisher RA, et al. Pseudo-partial moles: placental stem vessel hydrops and the association with Beckwith-Wiedemann syndrome and complete moles. Histopathology 2001; 39: 447-54.

6. Thaker HM. The partly molar pregnancy that is not a partial mole. Pediatr Dev Pathol 2005; 8: 146-7.

7. Sebire NJ, Fisher RA. Partly molar pregnancies that are not partial moles, additional possibilities and implications. Padiatr Dev Pathol 2005; 8: 732-3.

8. Parveen Z, Tongson Ignacio J, Fraser C, Killen J, Thompson K. Placental mesenchymal dysplasia. Arch Pathol Lab Med 2007; 131: 131-137.

9. Chen CP, Chern SR, Wang TY, Huang ZD, Huang MC, Chuang CY. Pregnancy with concomitant chorioangioma and placental vascular malformation with mesenchymal hyperplasia. Hum Reprod 2007; 22: 1114-1122.

10. Maher ER, Reik W. Beckwith-Wiedemann syndrome: imprinting in clusters revisited. J Clin Invest 2000; 105: 247-252.

11. Ohira S, Ookubo N, Kobara H, Tanaka K, Kikuchi N, Takatsu A, Ohya A. Placental Mesenchymal Dysplasia: Chronological Observation of Placental Images during Gestation and review of Literature. Gynaec Obstet Invest 2013; 75: 217-223.

12. Kaiser Rogers KA, McFadden DE, Livasy CA, Dansereau J, Jiang R, Knops JF, Lefebvre L, Rao KW, Robinson WP. Androgenetic/biparental mosaicism causes placental mesenchymal dysplasia. J Med Genet 2006; 43: 187-192.

13. Nayeri U. A, West A.B, H. K. Grossetta Nardini, J.A. Copel and A.K. Sfakianaki. Systemic review of sonographic findings of placental mesenchymal dysplasia and subsequent pregnancy outcome. Ultrasound Obstet Gynecol 2013; 41: 366-374.

\section{AUTHORS:}

1. Ramya T.

2. Uma Maheshwari G.

3. Chaitra V.

\section{PARTICULARS OF CONTRIBUTORS:}

1. Associate Professor, Department of Obstetrics \& Gynaecology, PSG Institute of Medical Sciences \& Research, Coimbatore, Tamil Nadu, India.

2. Associate Professor, Department of Pathology, PSG Institute of Medical Sciences \& Research, Coimbatore, Tamil Nadu, India.
3. Associate Professor, Department of Pathology, PSG Institute of Medical Sciences \& Research, Coimbatore, Tamil Nadu, India.

\section{NAME ADDRESS EMAIL ID OF THE CORRESPONDING AUTHOR:}

Dr. Ramya T, Associate Professor, Department of Obstetrics \& Gynaecology, PSG Institute of Medical Sciences \& Research, Coimbatore-641004,

Tamil Nadu, India.

Email: ramya.t2003@gmail.com

Date of Submission: 06/11/2014. Date of Peer Review: 07/11/2014. Date of Acceptance: 18/11/2014. Date of Publishing: 24/11/2014. 